\title{
Neuroanatomical findings of significant translational importance
}

\author{
Comments on: Holstein, G. R., Friedrich, V. L., and Martinelli, G. P. Exp Brain Res \\ (2016). doi:10.1007/s00221-016-4725-2
}

\author{
Ronald M. Harper ${ }^{1}$
}

Published online: 1 September 2016

(C) Springer-Verlag Berlin Heidelberg 2016

From time to time, a basic science article appears that transcends different fields and brings significant insights into a broad range of behavioral and clinical processes. Such translational importance is the case for a neuroanatomical study by Holstein and colleagues outlining the presence of imidazole-4-acetic acid-ribotide (IAARP) in neurons of the vestibulosympathetic pathway. The putative neurotransmitter also appears in other areas within the CNS, especially blood pressure regulatory areas, and particularly in the hippocampus. The demonstration here provides insights into processes, which may underlie multiple syndromes and conditions associated with sudden unexplained death, helps to explain mechanisms of action of common pharmaceutical means to lower blood pressure, and suggests processes by which widespread conditions, such as sleep-disordered breathing and epilepsy, can be accompanied by severe cardiovascular concerns.

The importance of central regulation of blood pressure in potentially fatal conditions is often overlooked, with a focus on dysfunction within an end organ, such as cardiac arrhythmia or failure of myocardial output, rather than attention to $\mathrm{CNS}$ processes responsible for maintaining vascular tone or providing appropriate sympathetic or parasympathetic drive to the myocardium. The awareness is further compromised by a directed focus to "conventional" baroreceptor-dorsal medullary-ventral medullary-spinal

This Editorial refers to the article available at doi: 10.1007/s00221-016-4725-2.

Ronald M. Harper

rharper@ucla.edu

1 Department of Neurobiology and the Brain Research Institute, David Geffen School of Medicine at UCLA, Los Angeles, CA 90095-1763, USA cord circuity, with little attention to vestibular/cerebellar pathways critical for maintaining postural and head adjustments to blood pressure, integrating respiratory action with momentary blood pressure changes, and dampening extremes of blood pressure changes. The Holstein et al.'s manuscript appropriately redirects attention to the multiple systems that can be disrupted by interference with the neurotransmitter distribution they revealed.

The literature is replete with clinical conditions which lead to sudden death, and which suspiciously appear to result from failure of the vestibulosympathetic system. One such condition, the sudden infant death syndrome (SIDS), is often accompanied by characteristics which share signs of marked sympathetic dysfunction similar to those found in shock, with profuse sweating and tachycardia, followed by profound bradycardia and hypotension with continued breathing. The triggers for that scenario and the processes by which autonomic processes are disrupted remain unclear, but a number of structural and functional characteristics related to support of sympathetic output appear in SIDS cases prior to the fatal event, including enhanced apoptosis of vestibular nuclei, maldevelopment of cerebellar neurons, and impaired responses to vestibular tilt tests. Other vestibular/cerebellar issues are present; the prone position significantly raises the risk for SIDS, and infants at risk for SIDS evaluated in the prone position in a tilt test show impaired blood pressure outcomes (Harper and Kinney 2010, Curr Pediatr Rev 6:39-47). The findings from the Holstein et al.'s manuscript raise the possibility that disturbances in a principal neurotransmitter/neuromodulator of the vestibulosympathetic system through structural injury by an asyet-undefined process, or by deleterious action on the neurotransmitter can establish circumstances that place control of sympathetic processes at risk, especially during development where redundant recovery systems may be immature. 
The potential for life-threatening blood pressure control concerns extends beyond the vestibular-cerebellum paths. The authors describe the high densities of IAARP immunopositive neurons in the hippocampus, the colocalization of IAARP and glutamate immunoflourescence, and the potential for IAARP binding to imidazoline receptors to suppress glutamatergic transmission. The hippocampal findings are also of importance for sudden death in infants and early childhood, since hippocampal maldevelopment has been described in such victims, and the hippocampus exerts significant roles in mediating forebrain regulation of blood pressure [Kinney et al. 2015, Acta Neuropathol 129(1):65-80]. However, other fatal scenarios may well develop from hippocampal injury, including sudden unexpected death in epilepsy (SUDEP) and the appearance of "dead in bed" phenomenon in diabetes. Ictal events in epilepsy patients can be followed by severe hypotension, a pattern which, if sustained, would lead to loss of perfusion to vital areas and death; interictal recordings show elevated blood pressure and diminished variability in heart rate (Nei et al. 2016, Epil Res 122:44-46). Both cerebellar and hippocampal injuries are common in people with epilepsy, a consequence of repeated excitotoxic injury, as well as the possibility of medication-induced damage. Diabetes is accompanied by severe injury in hippocampal, cerebellar, insular, medial prefrontal frontal cortex, and other blood pressure regulatory structures, and sudden unexpected death remains a significant concern in the condition. The presence of injury in those blood pressure regulatory structures raises the possibility of failed regulation of IAARP processes in a fatal hypotensive scenario.

Any description of blood pressure and sympathetic responses to manage routine body functions must consider several concerns, including those of physiological responses to altered central timing and laterality of neural influences. Delays in sympathetic outflow, or phaseshifting of centrally lateralized action raises the potential for late perfusion to transient challenges in body position or asymmetric sympathetic influences on cardiac ganglia, the latter a well-known recipe for establishing dangerous cardiac arrhythmia. Examples of such lateralized centrally delayed timing in ventral medullary blood pressure structures appear in conditions that elicit neurodegenerative damage, such as obstructive sleep apnea or congenital central hypoventilation syndrome (Harper et al. 2014, Prog Brain Res 209:275-293). Overall, timing delays or pathologically lateralized centrally impaired responses can result in abnormal outcomes, such as syncope on standing and mental confusion from failure to timely perfuse regional brain areas. The findings here provide at least one means to establish such timing distortions and asymmetric sympathetic output. Holstein et al. reflect on the need for rapid blood pressure responses to head or body movements, as outlined some time ago by Yates and colleagues [Yates 1992, Brain Res Rev, 17(1):51-59]. The authors then describe the colocalization of glutamate with IAARP receptors, a lateralized system design which allows rapid modulation of presympathetic neurons essential for the primary function of the vestibulocerebellum sympathetic pathway. However, that description also reveals mechanisms by which central neural sympathetic timing delays can be mediated. Targeted injury to either IAARP or glutamate neurotransmitter systems could significantly impair sympathetic outflow laterality or timing. Interference with either of the two neurotransmitter processes, or on the structures mediating the interactions between those systems would provide the basis for temporal distortions that could result in significant physiological consequences.

The implications of the findings for the use of common pharmaceutical means to reduce blood pressure are significant as well. In particular, the IAARP description addresses a mechanism by which clonidine and analogs reduce blood pressure, possibly by inhibiting RVLM parasympathetic neurons. Clonidine contains an imidazole ring, and binding for these agents using imidazoline receptor subtypes that are densely located in the medulla provide a process for hypotensive action for drugs containing a synthetic imidazoline ring.

The manuscript provides essential insights into mechanisms underlying vestibulocerebellar blood pressure management which are necessary to understand normal control of blood pressure. Of equal importance, the studies outline means by which a number of life-threatening outcomes can be mediated by processes which use the described neurotransmitter pathways, and the manner in which common pharmaceutical interventions operate. Those descriptions are valuable for the field, and provide an exceptional example of basic research with significant translational implications.

Acknowledgments Statement of support and COI: Dr. Harper is supported by NIH/NINDS UO1 NS090407

\section{Compliance with ethical standards}

Conflict of interest The author declares that there are no conflicts of interest. 